\title{
EXTENDED MILD-SLOPE EQUATION FOR RANDOM WAVES
}

\section{Changhoon Lee ${ }^{\mathrm{a}}$, Gunwoo Kim ${ }^{\mathrm{b}}$, Kyung-Duck Suh ${ }^{\mathrm{c}}$ and Yong-Sik Cho ${ }^{\mathrm{d}}$}

a Assistant Professor, Department of Civil and Environmental Engineering, Sejong Univ ersity, 98 Kunja-Dong, Kwangjin-Gu, Seoul 143-747, South Korea, Fax: +82-2-34083332,clee@sejong.ac.kr

${ }^{\mathrm{b}}$ Graduate Student, School of Civil, Urban, and Geosystem Engineering, Seoul National University, San 56-1, Shinlim-Dong, Gwanak-Gu, Seoul 151742, South Korea, maossi1@snu.ac.kr

${ }^{c}$ Professor, School of Civil, Urban, and Geosystem Engineering \& Research Institute of Marine Systems Engineering, Seoul National University, San 56-1, ShinlimDong, Gwanak-Gu, Seoul 151-742, South Korea, kdsuh@snu.ac.kr

${ }^{\mathrm{d}}$ Associate Professor, Department of Civil Engineering, Hanyang University, 17 Haengd ang-Dong, Songdong-Gu, Seoul 133-791, South Korea, ysc59@ hanyang.ac.kr

\section{Abstract}

A time-dependent extended mild-slope equation is derived from the elliptic equation of Chamberlain and Porter (1995) using the Taylor series technique. Numerical tests are made on a horizontally one-dimensional case for regular waves over sloping beds and for both regular and irregular waves over a ripple patch. Numerical results prove that the proposed model gives accurate results for both regular and irregular waves over rapidly varying topography. 
Keywords: Numerical model; Mild-

slope equation; Water waves; Random wave propagation

\section{Introduction}

Waves generated in deep water propagate to shallow water undergoing various forms of transformations, like shoaling, refraction, diffraction, and reflection, etc. Since the de velopment of $\quad$ a $\quad$ mildslope equation by Berkhoff (1972), many mathematical models have been developed to analyze the combined refraction and diffraction of waves. In order to model the propagat ion of random waves, time-dependent mildslope equations have also been developed. Smith and Sprinks (1975) developed a hyper bolic mildslope equation using Green's formula. Radder and Dingemans (1985) proposed a canoni cal form of the time-dependent mildslope equations based on the Hamiltonian theory of surface waves. Kubo et al. (1992) al so developed another type of time-dependent mildslope equation using the Taylor series expansion technique for waves with local frequen cies different from the carrier frequency. Lee and Kirby (1994) analyzed the mildslope equations developed by Smith and Sprinks, and Kubo et al. in terms of the dispers ion relation and energy transport. Considering the dispersion relation, Smith and Sprinks

' model is more accurate in shallower water $(k h \leq 0.2 \pi)$, whereas Kubo et al.'s model is 
more accurate in deeper water $(k h>0.2 \pi)$. Kubo et al.'s model is more accurate for en ergy transport but has a singularity problem in the higher frequency range.

Nishimura et al. (1983) developed hyperbolic mildslope equations by vertically integrating the continuity and momentum equations for lin ear waves. Starting from the time-dependent mildslope equation of Smith and Sprinks, Copeland (1985) developed similar hyperbolic equ ations using the characteristics of linear waves and the defined volume flux. The two mo dels of Nishimura et al. and Copeland predict the transformation of regular waves.

In the derivation of the mild-slope equations, an assumption of $|\nabla h| / k h<<1$ (where $\nabla=$ horizontal gradient operator, $k=$ wave number, and $h=$ water depth) was made so that the terms of the second-order bottom effect related to the bottom curvature and the square of the slope were neglected. To account for the effect of a steeply sloping and rapidly varying bottom topography, Massel (1993), and Chamberlain and Porter (1995) derived the extended mild-slope equation using the Galerkin-eigenfunction method. Chandrasekera and Cheung (1997) provided an alternative derivation of the extended equation based on the approach of Berkhoff. Suh et al. (1997) used the Green's second identity and Lagrangian formula to develop two ultimately equivalent hyperbolic equations for random waves. Lee et al. (1998) recast the elliptic formulation of Massel into a hyperbolic formulation, following the technique of Copeland.

In this study, by applying Kubo et al.'s method to the equation of Chamberlain and Po rter, we developed a time-dependent version of the extended mild- 
slope equation, which may be able to predict the transformation of random waves on rap idly varying topography. In order to verify the improved accuracy of our equation compa red to the equation of Kubo et al. in the case of a rapidly varying topography, the two $\mathrm{m}$ odels were initially applied to the problem of wave reflection from a planar slope with d ifferent inclinations (Booij, 1983). Subsequently, the equation was applied to the proble $\mathrm{m}$ of the resonant Bragg reflection of monochromatic waves from a ripple patch (Davies and Heathershaw, 1984). Finally, to investigate the applicability of the proposed equati on to random waves, numerical tests were performed on the transmission of unidirection al random waves normally incident to a finite ripple patch.

\section{Development of governing equation}

The extended mild-slope equation of Chamberlain and Porter (1995) is given by

$$
\nabla \cdot\left(C C_{g} \nabla \eta\right)+\left\{k^{2} C C_{g}+g u_{1} \nabla^{2} h+g u_{2}(\nabla h)^{2}\right\} \eta=0
$$

where the coefficients $u_{1}$ and $u_{2}$ determining the secondorder bottom effects are given by

$$
\begin{gathered}
u_{1}=\frac{\operatorname{sech}^{2} k h}{4(2 k h+\sinh 2 k h)}(\sinh 2 k h-2 k h \cosh 2 k h) \\
u_{2}=\frac{k \operatorname{sech}^{2} k h}{12(2 k h+\sinh 2 k h)^{3}}\left\{(2 k h)^{4}+4(2 k h)^{3} \sinh 2 k h-9 \sinh 2 k h \sinh 4 k h\right. \\
\left.+6 k h(2 k h+2 \sinh 2 k h)\left(\cosh ^{2} 2 k h-2 \cosh 2 k h+3\right)\right\}
\end{gathered}
$$


and $\eta$ is the water surface elevation, $C$ and $C_{g}$ are the phase speed and group velocity, respectively, of a wave with an angular frequency $\omega$, and $g$ is the gravitational accelera tion. The wave number $k$ is determined from the linear dispersion relation for Stokes $\mathrm{w}$ aves.

For random waves, the surface elevation is defined as the superposition of component waves

$$
\eta=\operatorname{Re}\left[\sum_{m=1}^{\infty} \eta_{m} e^{-i \omega_{m} t}\right]
$$

where $\omega_{m}$ and $\eta_{m}$ are the angular frequency and complex amplitude, respectively, of the $-m$-th component wave. In order to apply the extended mildslope equation to random waves, we define a variable $\hat{\eta}$ as

$$
\eta=\operatorname{Re}\left[\hat{\eta} e^{-i \bar{\omega} t}\right]
$$

where $\bar{\omega}$ is a carrier angular frequency of the random waves. From Eq. (4) and Eq. (5), we have

$$
\frac{\partial \hat{\eta}_{m}}{\partial t}=-i \Delta \omega_{m} \hat{\eta}_{m}
$$


where $\Delta \omega_{m}=\omega_{m}-\bar{\omega}$. The variable of component wave, $\hat{\eta}_{m}$, is a solution of Eq. (1), as well as $\eta_{m}$. Therefore, Eq. (1) can be rewritten in terms of $\hat{\eta}_{m}$. If we expand the quantiti es $\left(C C_{g}\right)_{m},\left(k^{2} C C_{g}\right)_{m},\left(u_{1}\right)_{m}$ and $\left(u_{2}\right)_{m}$ into the Taylor series of $\Delta \omega_{m}$ and use Eq. (6), we can obtain an equation in which all the variables can be determined by the coefficien ts of the carrier frequency only. Superposition of the resulting equations for all the comp onent waves yields the following equation

$$
\begin{aligned}
& \nabla \cdot\left(\bar{C} \bar{C}_{g} \nabla \hat{\eta}\right)+\left\{\bar{k}^{2} \bar{C} \bar{C}_{g}+g \bar{u}_{1} \nabla^{2} h+g \bar{u}_{2}(\nabla h)^{2}\right\} \hat{\eta}+i \nabla \cdot\left\{\frac{\overline{\partial\left(C C_{g}\right)}}{\partial \omega} \nabla \frac{\partial \hat{\eta}}{\partial t}\right\} \\
& +i\left\{\frac{\partial\left(k^{2} C C_{g}\right)}{\partial \omega}+g \frac{\overline{\partial u_{1}}}{\partial \omega} \nabla^{2} h+g \frac{\partial u_{2}}{\partial \omega}(\nabla h)^{2}\right\} \frac{\partial \hat{\eta}}{\partial t}=0
\end{aligned}
$$

where the over bar indicates the variables associated with the carrier angular frequency $\bar{\omega}$ and the coefficients are given by

$$
\begin{aligned}
\frac{\partial\left(C C_{g}\right)}{\partial \omega}=\frac{\bar{\omega}}{\bar{k}^{2}}\left[2(\bar{n}-1)+\frac{2 \bar{n}-1}{2 \bar{n}}\{1-(2 \bar{n}-1) \cosh 2 \bar{k} h\}\right] \\
\frac{\overline{\partial\left(k^{2} C C_{g}\right)}}{\partial \omega}=\bar{\omega}\left[2 \bar{n}+\frac{2 \bar{n}-1}{2 \bar{n}}\{1-(2 \bar{n}-1) \cosh 2 \bar{k} h\}\right] \\
\frac{\partial u_{1}}{\partial \omega}=-\frac{\bar{k} h \operatorname{sech}^{2} \bar{k} h}{2 \bar{\omega} \bar{n}(2 \bar{k} h+\sinh 2 \bar{k} h)}\{2 \bar{k} h \sinh 2 \bar{k} h \\
\left.+\left(\tanh \bar{k} h+\frac{1+\cosh 2 \bar{k} h}{2 \bar{k} h+\sinh 2 \bar{k} h}\right)(\sinh 2 \bar{k} h-2 \bar{k} h \cosh 2 \bar{k} h)\right\}
\end{aligned}
$$




$$
\begin{aligned}
\frac{\partial u_{2}}{\partial \omega}= & \frac{\bar{k} \operatorname{sech}^{2} \bar{k} h}{12 \bar{\omega} \bar{n}(2 \bar{k} h+\sinh 2 \bar{k} h)^{3}}\left[\left\{1-2 \bar{k} h \tanh \bar{k} h-\frac{6 \bar{k} h(1+\cosh 2 \bar{k} h)}{(2 \bar{k} h+\sinh 2 \bar{k} h)}\right\}\right. \\
& \times\left\{(2 \bar{k} h)^{4}+4(2 \bar{k} h)^{3} \sinh 2 \bar{k} h-9 \sinh 2 \bar{k} h \sinh 4 \bar{k} h\right. \\
& \left.+12 \bar{k} h(\bar{k} h+\sinh 2 \bar{k} h)\left(\cosh ^{2} 2 \bar{k} h-2 \cosh 2 \bar{k} h+3\right)\right\} \\
& +\bar{k} h\left\{8(2 \bar{k} h)^{3}+24(2 \bar{k} h)^{2} \sinh 2 \bar{k} h+8(2 \bar{k} h)^{3} \cosh 2 \bar{k} h\right. \\
& -18 \cosh 2 \bar{k} h \sinh 4 \bar{k} h-36 \sinh 2 \bar{k} h \cosh 4 \bar{k} h \\
& +12(2 \bar{k} h+\sinh 2 \bar{k} h+2 \bar{k} h \cosh 2 \bar{k} h)\left(\cosh { }^{2} 2 \bar{k} h-\cosh 2 \bar{k} h+3\right) \\
& +48 \bar{k} h \sinh 2 \bar{k} h(\bar{k} h+\sinh 2 \bar{k} h)(\cosh 2 \bar{k} h-1)\}] \\
\bar{n} & =\frac{\bar{C}}{\bar{C}}=\frac{1}{2}\left(1+\frac{2 \bar{k} h}{\sinh 2 \bar{k} h}\right)
\end{aligned}
$$

Eq. (7) is a time-dependent extended mildslope equation for random waves. If $\bar{u}_{1}=\bar{u}_{2}=0$, Eq. (7) reduces to the equation of Kub o et al. (1992).

\section{$\underline{\text { 3. Comparison of the proposed equation with Suh et al.'s equation }}$}

The proposed equation and Suh et al.'s (1997) equation are known to accurately predi ct waves on rapidly varying topography. The accuracy of the equations is due to the incl usion of the second-order bottom effect terms. In this section, the terms of higherorder bottom effect are compared between the two equations. The two equations are also $\underline{\text { known to be capable of predicting transformation of random waves with a narrow frequ }}$ ency band. So, the applicability of the two models to random waves is investigated in ter ms of the dispersion relation following the geometric optics approach of Lee and Kirby ( 
Using the relation of $\partial \tilde{\phi} / \partial t=-g \eta$, where $\tilde{\phi}$ is the velocity potential at the mean wa ter level, the equation of Suh et al. can be written in terms of $\eta \underline{\text { as: }}$

$$
\underline{\frac{\partial^{2} \eta}{\partial t^{2}}-\nabla \cdot\left(\bar{C} \bar{C}_{g} \nabla \eta\right)+\left(\bar{\omega}^{2}-\bar{k}^{2} \bar{C} \bar{C}_{g}\right) \eta+\bar{\omega}^{2}\left(\bar{R}_{1}(\nabla h)^{2}+\bar{R}_{2} \nabla^{2} h\right) \eta=0}
$$

where the parameters $\bar{\omega}^{2} \bar{R}_{1}$ and $\bar{\omega}^{2} \bar{R}_{2}$ determining the secondorder bottom effects are mathematically equivalent to $-g \bar{u}_{2}$ and $-g \bar{u}_{1}$, respectively, of the proposed equation (7). These higherorder bottom effects were studied in detail by Lee et al. (1998).

The geometric optics approach is used to get the dispersion relations of the proposed $\underline{\text { equation and Suh et al.'s equation. Let the surface elevation }} \eta \underline{\text { be }}$

$$
\eta=a(x, y) \exp (i \psi)
$$

where $a(x, y)$ is a complex amplitude which modulates in space, and the phase function $-\underline{\psi} \underline{\text { has the following relation with the local wave number }} \underline{k} \underline{\text { and angular frequency }} \underline{\omega} \underline{\mathrm{a}}$ $\underline{\mathrm{s}:}$ 


$$
k=\nabla \psi, \quad \omega=-\frac{\partial \psi}{\partial t}
$$

Then the variable $\hat{\eta}$ in Eq. (7) may be written as:

$$
\hat{\eta}=a(x, y) \exp [i(\psi+\bar{\omega} t)]
$$

Substituting Eq. (16) into Eq. (7) and neglecting both the higherorder bottom effects and diffraction effects give, the real part gives the dispersion relati on:

$$
\frac{k}{\bar{k}}=\sqrt{1+\frac{2\left(\frac{\omega}{\bar{\omega}}-1\right)}{\overline{\frac{\bar{C}_{g}}{\bar{C}}+\left(\frac{\omega}{\bar{\omega}}-1\right) \frac{\bar{\omega}}{\bar{C}^{2}} \frac{\partial}{\partial \omega}\left(C C_{g}\right)}}}
$$

On the other hand, substituting Eq. (14) into the Suh et al.'s equation (13) and again neg lecting those effects, the real part gives the dispersion relation:

$$
\frac{k}{\bar{k}}=\sqrt{1+\frac{\bar{C}}{\bar{C}_{g}}\left[\left(\frac{\omega}{\bar{\omega}}\right)^{2}-1\right]}
$$

These dispersion relations can be compared with the exact one for a linear wave: 


$$
\frac{k}{\bar{k}}=\left(\frac{\omega}{\bar{\omega}}\right)^{2} \frac{\tanh \bar{k} h}{\tanh k h}
$$

These dispersion relations are dependent on the relative wave depth $(\bar{k} h)$ correspondi ng to the carrier frequency. Fig. 1 shows the dispersion relations in a shallow water ( $\bar{k} h=0.05 \pi)$ and in a deep water $(\bar{k} h=2 \pi)$. In the case of shallow water, the dispersion relation of the proposed model is close to the exact one at higher frequencies but diverg es at lower frequencies. However, the agreement between the dispersion relation of Suh et al.'s model and the exact one is excellent. For deep water, the dispersion relation of th e proposed model is closer to the exact one than Suh et al.'s model at lower frequencies. However, the dispersion relation of Suh et al.'s model is closer to the exact one at highe $\underline{\mathrm{r} \text { frequencies. }}$

The relative water depth is obtained such that there is a minimal squared relative error in the dispersion relation of the proposed model compared to Suh et al.'s model. The sq uared relative error is defined as:

$$
E(\bar{k} h)=\int_{\frac{\omega}{\bar{\omega}}=0.2}^{1.8}\left(\frac{k_{s}-k_{p}}{k_{s}}\right)^{2} d\left(\frac{\omega}{\bar{\omega}}\right)
$$

where $k_{s}$ is the wave number obtained by Eq. (18) and $k_{p}$ is the wave number obtained by Eq. (17). The squared relative errors are found in the depth range of $\bar{k} h=0.05 \pi \sim 1.00 \pi$. At the relative water depth of $\bar{k} h=0.247 \pi$ the squared relative err 
or is minimized, i.e. the dispersion relations of the two models are almost equal to each other. Fig. 2 shows the dispersion relations when the relative water depth is $0.247 \pi$. Th e two dispersion relations are almost equal to each other except in the higher frequency $r$ ange.

For a linear dispersion, Suh et al.'s model is more accurate than the proposed model $\underline{\text { when the relative water depth is shallower than } 0.247} \pi$. When the relative water depth $\mathrm{i}$ s deeper than $0.247 \pi$, the proposed model shows better accuracy at lower frequencies, a $\underline{\text { nd Suh et al.'s model is more accurate at higher frequencies. }}$

In shallow water, the non-
linearity of waves becomes prominent and thus both the proposed model and Suh et al.'s $\underline{\text { model, which are linear, cannot yield accurate solutions for the dispersion relation. In re }}$ ality, the incident wave spectrum has little energy at higher frequencies, and most of the wave energy is in the lower frequency range. Therefore, the accuracy of the dispersion $r$ elation at lower frequencies determines the accuracy of the major change of a wave ener gy spectrum. This tendency gives an advantage to the proposed model compared to Suh et al.'s model.

\section{Numerical experiments}

To examine the accuracy of the proposed model, we conducted numerical experimen ts with both the proposed model and Kubo et al.'s (1992) model for monochromatic wa 
ves propagating over a planar slope using different inclinations of Booij (1983). The refl ection coefficients calculated by the models were compared against those calculated by $\mathrm{t}$ he finite element method (Suh et al., 1997). The two models were also applied to the cas e of a wave propagating over the ripple patch of Davies and Heathershaw (1984). The ca lculated reflection coefficients were compared against the experimental data. Finally, th e two models and the model of Suh et al. (1997) were tested for random waves propagat ing over the ripple patch. The solutions were compared against the finite element model solution, which can be regarded as an exact solution for linear random waves.

\subsection{Finite difference method}

Internal generation of waves has been used widely in generating waves in timedependent wave equations. Using the viewpoint of mass transport Larsen and Dancy (1983) employed a line source to generate waves in the staggered-grid solution of the Boussinesq equations. Lee and Suh (1998), and Lee et al. (2001) found that the internal generation of waves would be more accurate using the viewpoint of energy transport. However, the line source method may cause a problem for un-staggered grid systems. For this reason, Wei et al. (1999) generated waves using the source function method in the un-staggered grid solution of the Boussinesq equations. It is not possible to get a staggered grid solution for the proposed model. Therefore, we adopted a source function method to generate the waves.

Sponge layers were placed at the outside boundaries to remove wave reflection from $\mathrm{t}$ he boundaries by dissipating wave energy inside the sponge layers. The proposed model 
equation (7) is therefore modified as:

$$
\begin{aligned}
& \nabla \cdot\left(\bar{C} \bar{C}_{g} \nabla \hat{\eta}\right)+\left\{\bar{k}^{2} \bar{C} \bar{C}_{g}+g \bar{u}_{1} \nabla^{2} h+g \bar{u}_{2}(\nabla h)^{2}\right\}\left(1+i \frac{D_{s}}{\bar{n}}\right) \hat{\eta} \\
& +i \nabla \cdot\left\{\frac{\overline{\partial\left(C C_{g}\right)}}{\partial \omega} \nabla \frac{\partial \hat{\eta}}{\partial t}\right\} \\
& +i\left\{\frac{\overline{\partial\left(k^{2} C C_{g}\right)}}{\partial \omega}+g \frac{\overline{\partial u_{1}}}{\partial \omega} \nabla^{2} h+g \frac{\overline{\partial u_{2}}}{\partial \omega}(\nabla h)^{2}\right\}\left(1+i \frac{D_{s}}{\bar{n}}\right) \frac{\partial \hat{\eta}}{\partial t}=f(x, y, t)
\end{aligned}
$$

where $f(x, y, t)$ is a source function and the damping coefficient $D_{s}$ is given by

$$
D_{s}=\left\{\begin{array}{lr}
0, & \text { outside sponge layer } \\
\frac{\exp (d / S)-1}{\exp (1)-1}, & \text { inside sponge layer }
\end{array}\right.
$$

where $d$ is the distance from the starting point of the sponge layer and $S$ is the thicknes s of the sponge layer. In a dimensional domain, the modified model is discretized by the CrankNicolson method in time and space. Initially, surface elevations are set to zero. In order t o generate waves gradually, the source function is multiplied by $\tanh (t / p \bar{T})$ where $\bar{T} \mathrm{i}$ $\mathrm{s}$ the carrier wave period and $p$ is a parameter corresponding to the rate of slow wave $\mathrm{g}$ eneration. Since the sponge layer reduces the incoming wave energy effectively, the refl ective condition at the boundaries can be used.

\subsection{Wave reflection of monochromatic waves from a planar slope}


We conducted numerical experiments with both the proposed model and Kubo et al.'s model for monochromatic waves propagating over a planar slope, each end of which was connected to a constant-depth region, as tested by Booij (1983) who investigated the accuracy of the mild-slope equation with respect to bottom slope. Since Booij's tests, many researchers have adopted this numerical experiment to demonstrate accuracy of their model. The computational domain for the numerical test is shown in Fig. 3. The water depths on the up-wave and down-wave sides of the slope are $h_{1}=0.6 \mathrm{~m}$ and $h_{2}=$ $0.2 \mathrm{~m}$, respectively, and the width of the slope, $b$, is varied so that the inclination of the slope varies. The wave period is $2 \mathrm{~s}$. The grid size used is $\Delta x=0.05 \mathrm{~m}$, and the time step is $\Delta t=0.05 \mathrm{~s}$.

Fig. 4 shows the reflection coefficients calculated by the proposed equation, the Kubo et al.'s equation, and the finite element model of Suh et al. The finite element model sol

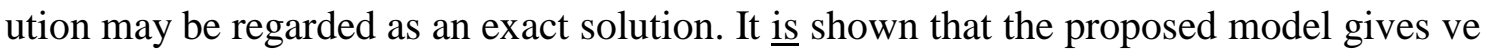
ry close answers to those of the finite element model and the reflection coefficient beco mes stable even for very steep slopes. Conversely, Kubo et al.'s model underpredicts the reflection coefficient for steeper slopes. Even for very mild slopes, the prop osed model, and Kubo et al.'s model show some differences and the results of the finite element model coincide with those of the proposed model rather than the Kubo et al.'s model.

Recently, Lee et al. (1998) indicated that bed topography is not sufficient to determine the accuracy of a wave equation with respect to the bottom slope, because the 
water depth tested is only in water of immediate depth and the two slope discontinuities affect the solution. The slope discontinuities are taken into account in the extended-mild slope equation through the bottom curvature terms.

\subsection{Bragg reflection of monochromatic waves from a ripple patch}

When surface waves are normally incident over a ripple patch, a significant portion of the wave energy is reflected from the ripple patch if the wavelength of the surface wave is twice that of the ripple. This wave reflection is called the resonant Bragg reflection.

Davies and Heathershaw reported a series of experimental data for different numbers of ripples and water depths. In their experiment, the ripple wavelength and amplitude w ere $1 \mathrm{~m}$ and $5 \mathrm{~cm}$, respectively, and the numbers of ripples were 2,4 , and 10 . The water

depth in the constantdepth region was $15.6 \mathrm{~cm}$ for the case of 2 and 4 ripples and $31.3 \mathrm{~cm}$ for the case of $10 \mathrm{r}$ ipples. This experimental data has been used for comparison with various numerical mo dels by a number of researchers. All of them showed that the mildslope equation gives a good agreement with the experimental data for the cases of 2 and 4 ripples, but for the case of 10 ripples, it fails to predict the magnitude of the Bragg refl ection. Therefore, in this study, a numerical test was made only for the case of 10 ripples . The water depth in the computational domain is given by 


$$
h(x)=\left\{\begin{array}{lr}
h_{c,} & x \leq x_{r} \\
h_{c}-A \sin \left[K\left(x-x_{r}\right)\right], & x_{r} \leq x \leq x_{r}+n L_{r} \\
h_{c,} & x \geq x_{r}+n L_{r}
\end{array}\right.
$$

where $A$ is the ripple amplitude, $L_{r}(=2 \pi / K)$ is the ripple wavelength, $n$ is the number of ripples, $h_{c}$ is the water depth in the constantdepth region, $K$ is the wave number of the ripple, and $x_{r}$ is the $x$ coordinate of the starting point of the ripple patch. The grid size used is $\Delta x=L / 30$ and $\Delta t=T / 30$, where $L \underline{\text { is the wavelength and period, respectively, of the surface waves. } \mathrm{T}}$ he computational domain for the numerical test is shown in Fig. 5 where $L_{\max }$ is the wav elength on a flat bottom satisfying $2 k / K=0.5$.

Reflection coefficients calculated according to the proposed model and Kubo et al.'s

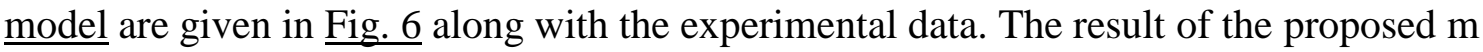
odel describes the resonant peak very well. However, the model of Kubo et al., while co rectly positioning the resonant reflection, completely fails to predict its magnitude.

\subsection{Transmission of random waves over a ripple patch}

Linear dispersion properties of the type of the proposed model were verified by Kubo et al. (1992), who simulated the propagation of wave groups using their equation. Suh et al. (1997) made numerical tests for the transmission of unidirectional random waves ov er the ripple patch of Davies and Heathershaw (1984). In this study, we also conducted t he same numerical tests. 
water spectrum was used as a target spectrum (Bouws et al., 1985):

$$
S(f)=\alpha g^{2}(2 \pi)^{-4} f^{-5} \exp \left[-1.25\left(f / f_{p}\right)^{-4}\right] \gamma^{\exp \left[-\left(f / f_{p}-1\right)^{2} / 2 \sigma^{2}\right]} \phi_{k}(f, h)
$$

$\underline{\text { where }} \underline{\alpha} \underline{\text { is a spectral parameter, }} \underline{\gamma} \underline{\text { is the peak enhancement factor, }} \underline{\sigma} \underline{\text { is the spectral wi }}$ dth parameter, and the Kitaigordskii shape function, $\phi_{k}(f, h) \underline{\text { incorporates the effect of } \mathrm{t}}$ he finite water depth. Two cases of numerical tests were conducted: one with a narrow $\mathrm{f}$ requency spectrum $(\gamma=20)$ and the other with a broad frequency spectrum $(\gamma=2)$. $\alpha=7.57 \times 10^{-4}$, and the peak frequency of input wave, $f_{p}=0.767 \mathrm{~Hz}$ were used in both cases. The computational domain was same as Fig. 5 , where $L_{\max }$ was selected as the $\mathrm{w}$ avelength corresponding to the lowest frequency, and the width of sponge layer was 2.5 $L_{\max }$. At the peak frequency of $f_{p}=0.767 \mathrm{~Hz}, 2 k_{p} / K$ is 1.0 and thus significant wave reflection from the ripple was expected near the peak frequency.

For the narrow spectrum case, the input spectrum was confined between 0.67 and 1.0 $0 \mathrm{~Hz}$, and for the broad spectrum case, the input spectrum was confined between 0.59 an d $1.63 \mathrm{~Hz}$. The carrier frequency $\bar{f}$ was selected as $0.79 \mathrm{~Hz}$ for the narrowbanded spectrum and $0.98 \underline{\mathrm{Hz}}$ for the broad-banded spectrum by weightaveraging the input spectrum. In solving Eq. (7), the wave parameters were chosen to be the values corresponding to the carrier frequency. The water surface elevation was recor 
ded at a distance $1.5 L_{\max }$ down-wave from the end of the ripple patch.

Fig. 7 shows a comparison of the transmitted wave spectra calculated by the proposed model and Kubo et al.'s model, against the finite element solution for the case of a narr ow-

banded frequency spectrum. As expected, the transmitted wave spectrum shows a signifi cant reduction compared with the input spectrum near the Bragg resonant peak, whereas most of the wave energy is transmitted over the ripples for the wave components whose frequency is far from the Bragg resonant frequency. Notably, the solution by Kubo et al. 's model is far from the finite element solution, predicting a much larger wave transmiss ion than the solution of the proposed model or the finite element solution. These results are nearly the same as those of Suh et al.'s model.

Fig. 8 shows a similar comparison for the case of a broadbanded frequency spectrum. As with the case of the narrowbanded spectrum, a reduction of the transmitted spectrum is observable near the Bragg $r$ esonant peak, and the transmitted spectrum is in good agreement with the finite element solution. The solution of the Kubo et al.'s model differs markedly from the finite eleme nt solution.

Suh et al. (1997) reported that accurate results could be obtained by dividing the frequ ency range into several bands and by modeling each of them with a representative carrie $\mathrm{r}$ frequency. In particular, use of a single carrier frequency in the case of a broadbanded spectrum was not sufficient to obtain a reasonably accurate result. Fig. 9 shows 
a comparison of the transmitted wave spectra calculated by the proposed model and the model of Suh et al. against the finite element solution for the case of a broadbanded frequency spectrum when a single band spectrum is used. In contrast to the prop osed model, the model of Suh et al. was not able to predict the reduction of the transmitt ed spectrum.

Fig. 10 shows the dispersion relations of Suh et al.'s model and the proposed model a gainst the linear wave in the case of the broadbanded spectrum $(\bar{k} h=0.438 \pi)$. In the lower frequency range, the wave number of the proposed model is close to the exact one, whereas the wave number of Suh et al.'s mode 1 is smaller than the exact one and even diverges to negative infinity. On the other hand, in the higher frequency range, the wave number of Suh et al.'s model is more accurate $t$ han the proposed model and the wave number of the proposed model diverges to positiv e infinity. Because the dispersion relation of Suh et al.'s model does not agree with the e xact one in lower frequency range, the Bragg reflection in lower frequency range cannot be predicted accurately whereas the proposed model does predict this reflection accurate ly.

\section{Conclusions}

A

time-

dependent wave model has been developed for waves propagating over rapidly varying $\mathrm{t}$

opography based on the mildslope equation of Chamberlain and Porter (1995). Without the higher- 
order bottom effect terms, the resulting equation reduces to the time-dependent mildslope equation developed by Kubo et al. (1992).

The proposed equation and Suh et al.'s (1997) equation were compared analytically in terms of linear dispersion. Suh et al.'s model is more accurate than the proposed model at a relative water depth shallower than $0.247 \pi$. When the relative water depth is deepe $\underline{\mathrm{r} \text { than } 0.247} \pi$, the proposed model is more accurate in the lower frequency range, and $\mathrm{S}$ $\underline{\text { uh et al.'s model is more accurate in the higher frequency range. }}$

Tests were made for the monochromatic wave reflections from a planar slope and peri odic ripples. The results of the proposed model and Kubo et al.'s model were compared with those from the finite element method or experimental data. Of all the cases tested, $t$ he proposed model gave accurate results, whereas Kubo et al.'s model failed to predict $\mathrm{t}$ he major characteristics of the phenomenon.

$\underline{\text { Tests were also made for the transmission of unidirectional random waves normally } \mathrm{i}}$ ncident on a finite ripple patch. For both narrow and broad spectra, the solutions of the $p$ roposed model and Kubo et al.'s model were compared against the finite element solutio $\underline{\mathrm{n}}$. The comparisons demonstrated an improved accuracy of the proposed model than $\mathrm{Ku}$ bo et al.'s model. For a broad spectrum, comparisons were made between the solutions of the proposed model and Suh et al.'s model. Using a single weightaveraged frequency as a carrier frequency, the proposed model gave more accurate solut ions. 


\section{Acknowedgements}

This first author wishes to acknowledge the financial support of the Korea Science and

$\begin{array}{llll}\text { Engineering } & \text { Foundation } & \text { (No. } & \text { R01-2000-000-00365- }\end{array}$

$0)$. The second and third authors wish to acknowledge the financial support of the Brain

Korea 21 Project. The fourth author wishes to acknowledge the financial support of Nati onal Research Laboratory Program (Coastal Engineering Laboratory, Hanyang Universit y)

\section{References}

Berkhoff, J.C.W., 1972. Computation of combined refraction-diffraction. Proc. 13th Coastal Eng. Conf., ASCE, 471-490.

Booij, N., 1983. A note on the accuracy of the mildslope equation. Coastal Eng., 7, 191-203.

Bouws, E., Günther, H., Rosenthal, W., Vincent, C.L., 1985. Similarity of the wind wav e spectrum in finite depth 1. Spectral form. J. Geophys. Res., 90(C1), 975-986.

Chamberlain, P.G., Porter, D., 1995. The modified mild-slope equation. J. Fluid Mech., 291, 393-407.

Chandrasekera, C.N., Cheung, K.F., 1997. Extended linear refraction-diffraction model. J. Waterw. Port Coastal Ocean Eng., 123, 280-296. 
Copeland, G.J.M., 1985. A practical alternative to the mild-slope wave equation. Coastal Eng., 9, 125-149.

Davies, A.G., Heathershaw, A.D., $1984 . \quad$ Surfacewave propagation over sinusoidally varying topography. J. Fluid Mech., 144, 419-443. Kubo, Y., Kotake, Y., Isobe, M., Watanabe, A., 1992. Time-dependent mild slope equation for random waves. Proc. 23rd Coastal Eng. Conf., ASCE, 419-431.

Larsen, J., Dancy, H., 1983. Open boundaries in short wave simulations a new approach. Coastal Eng., 7, 285-297.

Lee, $\quad$ C., $\quad$ Cho, $\quad$ Y.-

S., Yum, K., 2001. Internal generation of waves for extended Boussinesq equations.

Coastal. Eng., 42, 155-162.

Lee, C., Kirby, J.T., 1994. Analytical comparison of time-dependent mildslope equations. J. Korean Society of Coastal and Ocean Engrs., 6, 389-396.

Lee, C., Park, W.S., Cho, Y.-S., Suh, K.D., 1998. Hyperbolic mild-slope equations extended to account for rapidly varying topography. Coastal Eng., 34, 243-257.

Lee, C., Suh, K.D., 1998. Internal generation of waves for time-dependent mildslope equations. Coastal Eng., 34, 35-57.

Massel, $\quad$ S.R., $1993 . \quad$ Extended refractiondiffraction equation for surface waves. Coastal Eng., 19, 97-126.

Nishimura, H., Maruyama, K., Hirakuchi, H., 1983. Wave field analysis by finite differe nce method. Proc. 30th Japanese Conf. Coastal Eng., 123-127 (in Japanese).

Porter, D., Staziker, D.J., 1995. Extension of the mildslope equation. J. Fluid Mech., 300, 367-382.

Radder, A.C., Dingemans, M.W., 1985. Canonical equations for almost periodic, weakly 
nonlinear gravity waves. Wave Motion, 7, 473-485.

Smith, R., Sprinks, T., 1975. Scattering of surface waves by a conical island. J. Fluid Me ch., 72, 373-384.

Suh, $\quad$ K.D., $\quad$ Lee, $\quad$ C., $\quad$ Park, $\quad$ W.S., $1997 . \quad$ Timedependent equations for wave propagation on rapidly varying topography. Coastal En g., 32, 91-117.

Wei, G., Kirby, J.T., Sinha, A., 1999. Generation of waves in Boussinesq models using a source function method. Coastal. Eng., 36, 271-299.

\section{Captions of figures}

Fig. 1 Dispersion relation for (a) $\bar{k} h=0.05 \pi$ and (b) $\bar{k} h=2.0 \pi$; solid line $=\underline{\text { exact}}$, dash ed line $=$ proposed model, dash-dotted line $=$ Suh et al.'s model.

Fig. 2 Dispersion relation for $\bar{k} h=0.247 \pi$; solid line $=\underline{\text { exact}}$, dashed line $=$ proposed $\mathrm{m}$ odel, dash-dotted line $=$ Suh et al.'s model.

Fig. 3 Computational domain for numerical test of waves propagating over a planar slop e. 
Fig. 4 Reflection coefficient vs. width of a plane slope; dashed line= Kubo et al.'s mode 1, solid line $=$ proposed model, circle $=$ finite element model.

Fig. 5 Computational domain for numerical test of waves propagating over a ripple patc h.

Fig. 6 Comparison of solutions of proposed model and Kubo et al.'s model, against exp erimental data of Davies and Heathershaw (1984); solid line = proposed model, dashed 1 ine $=$ Kubo et al.'s model, filled circle $=$ experimental data.

Fig. 7 Incident narrow-banded spectrum and transmitted spectra calculated by time$\begin{array}{llll}\text { dependent with } & \text { equations }\end{array}$ averaged carrier frequency; short dashed line $=$ incident wave, solid line $=$ finite element model, long dashed line $=$ proposed model, dash-dotted line $=$ Kubo et al.'s model .

Fig. 8 Same as Fig. 7, but for broad-banded spectrum.

Fig. 9 Incident broad-banded spectrum and transmitted spectra calculated by timedependent equations with weight-averaged carrier frequency; short dashed line = incident wave, solid line $=$ finite element model, long dashed line $=$ proposed model, dash-dotted line $=$ Suh et al.'s model.

Fig. 10 Dispersion relation for $\bar{k} h=0.438 \pi$; solid line $=\underline{\text { exact}}$, dashed line $=$ proposed 
model, dash-dotted line $=$ Suh et al.'s model .

(a)

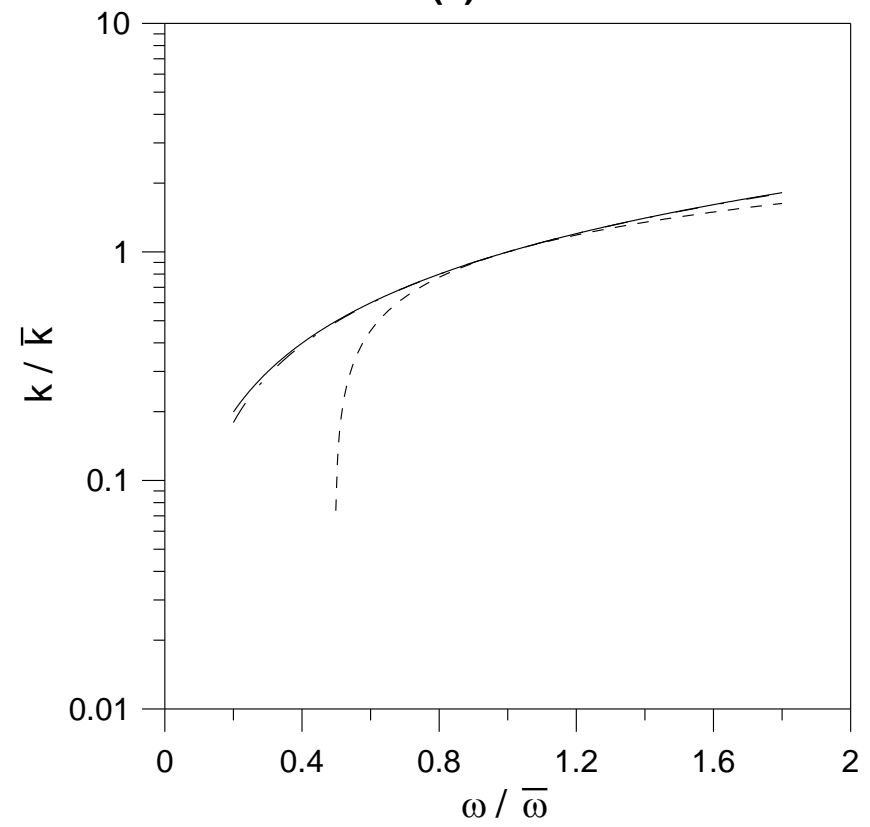


(b)

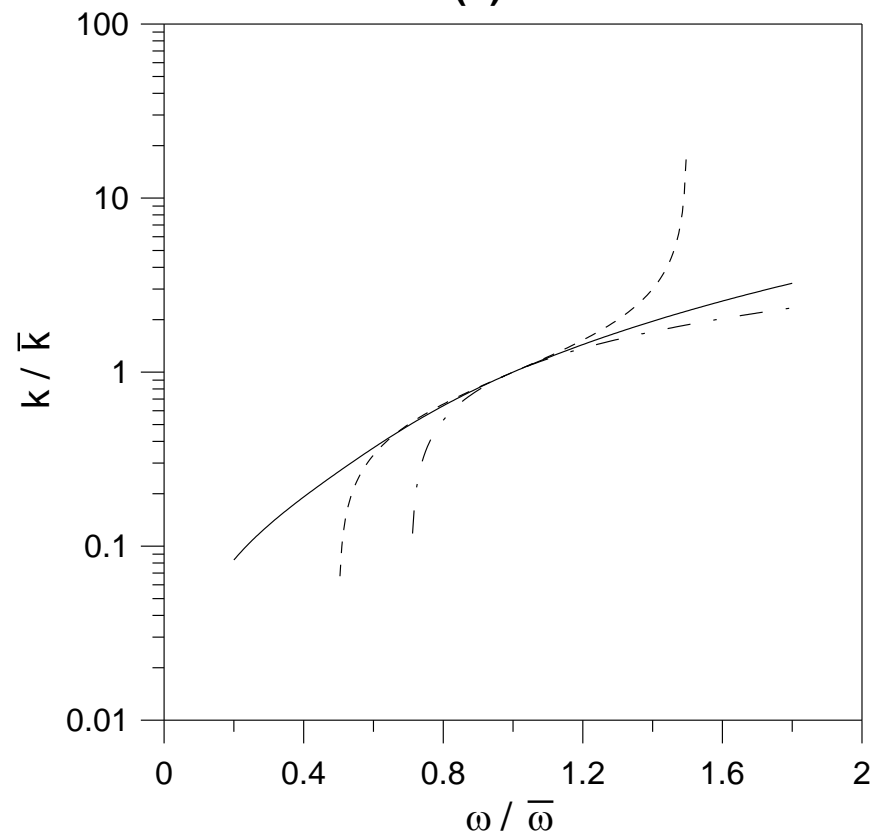

Fig. 1 Dispersion relation for (a) $\bar{k} h=0.05 \pi$ and (b) $\bar{k} h=2.0 \pi$; solid line $=$ exact, dashed line $=$ proposed model, dash-dotted line $=$ Suh et al.'s model.

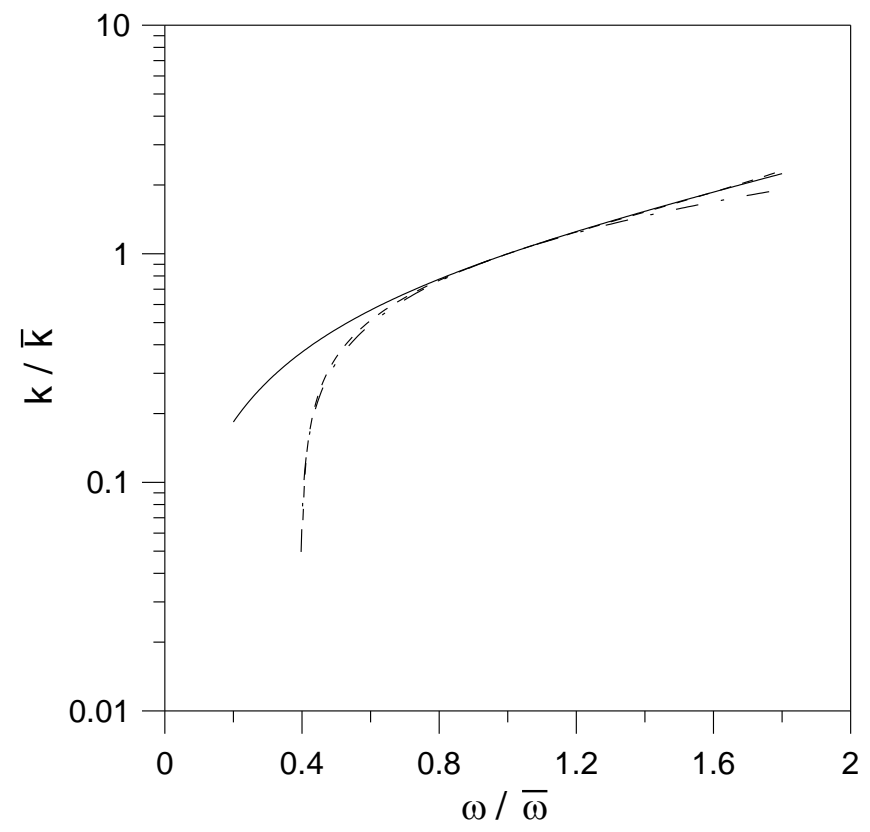

Fig. 2 Dispersion relation for $\bar{k} h=0.247 \pi$; solid line $=$ exact, dashed line $=$ proposed $\mathrm{m}$ 
odel, dash-dotted line $=$ Suh et al.'s model.

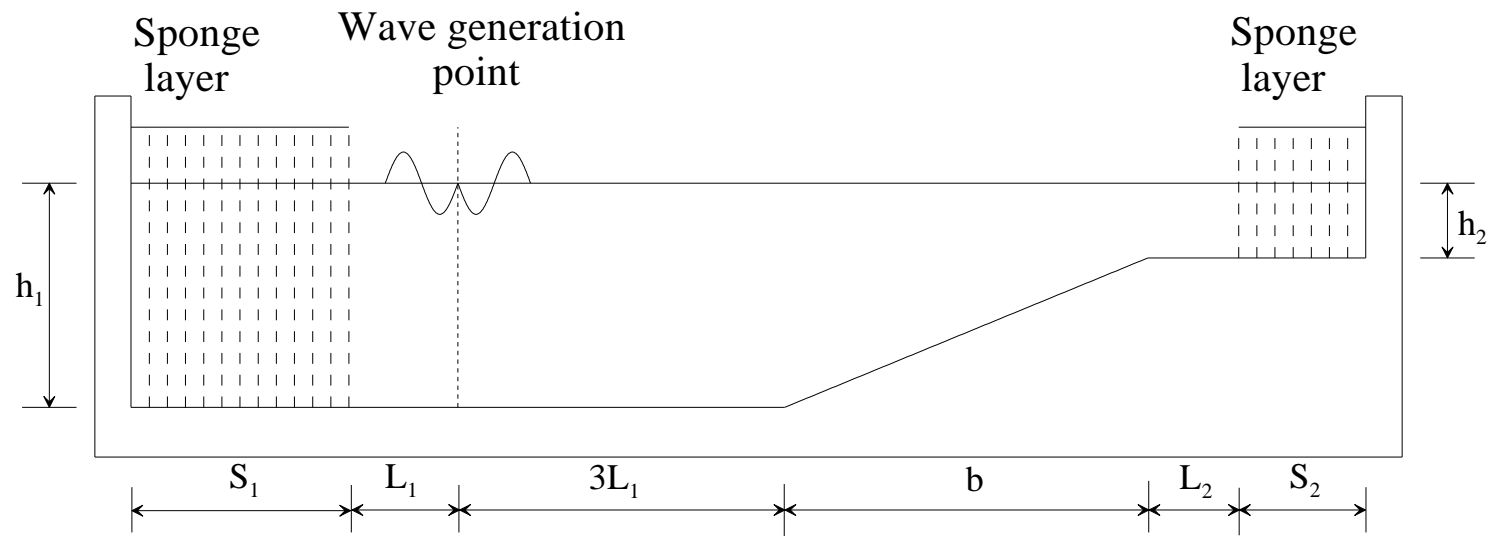

Fig. 3 Computational domain for numerical test of waves propagating over a planar slop e.

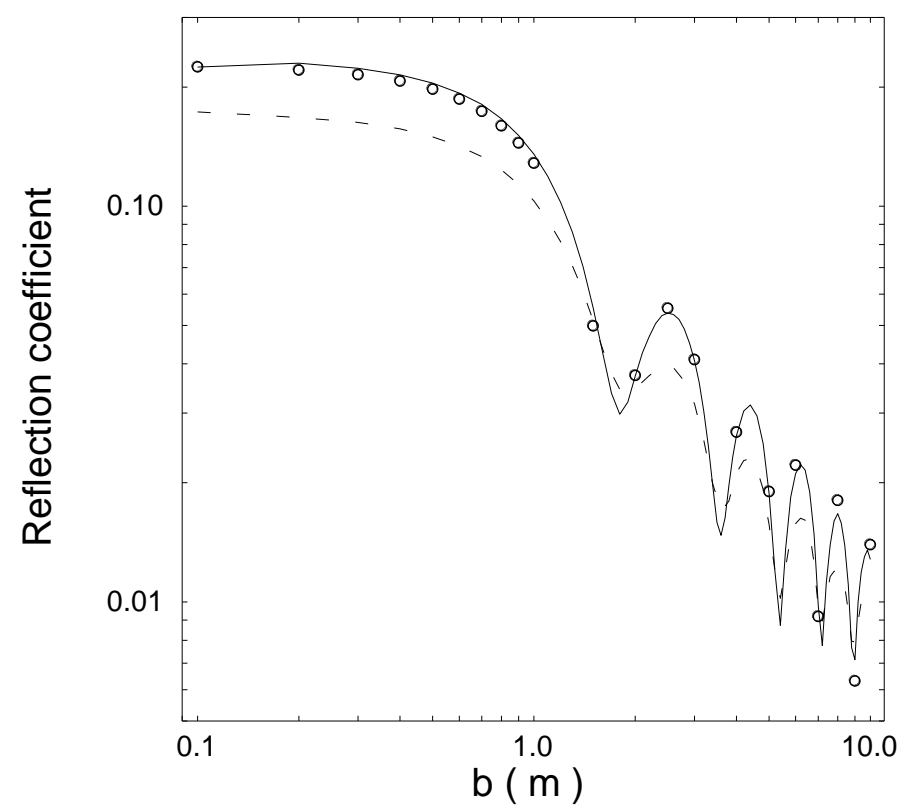


Fig. 4 Reflection coefficient vs. width of a plane slope; dashed line= Kubo et al.'s mode 1 , solid line $=$ proposed model, circle $=$ finite element model.

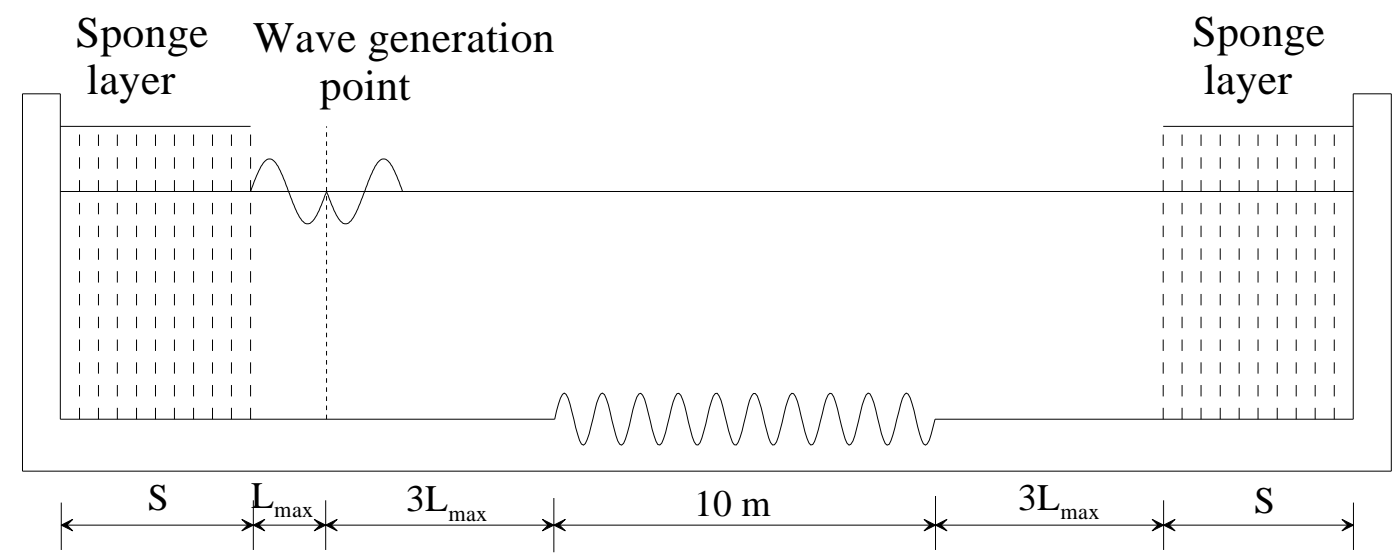

Fig. 5 Computational domain for numerical test of waves propagating over a ripple patc h.

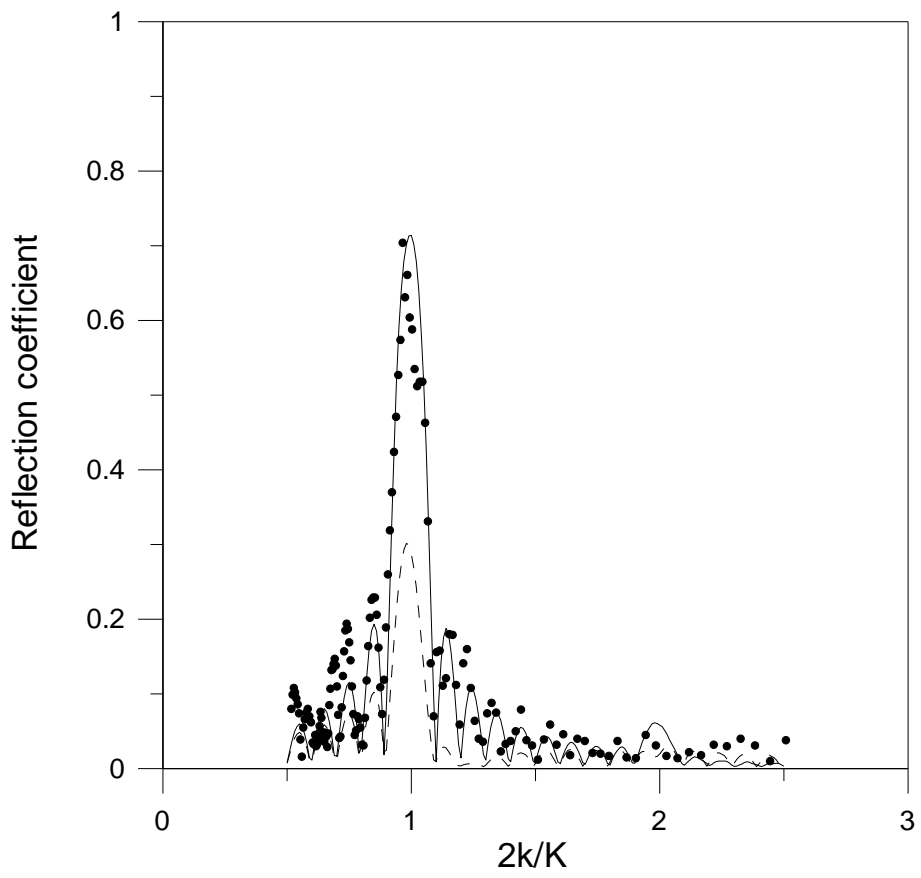

Fig. 6 Comparison of solutions of proposed model and Kubo et al.'s model, against exp erimental data of Davies and Heathershaw (1984); solid line = proposed model, dashed 1 
ine $=$ Kubo et al.'s model, filled circle $=$ experimental data.

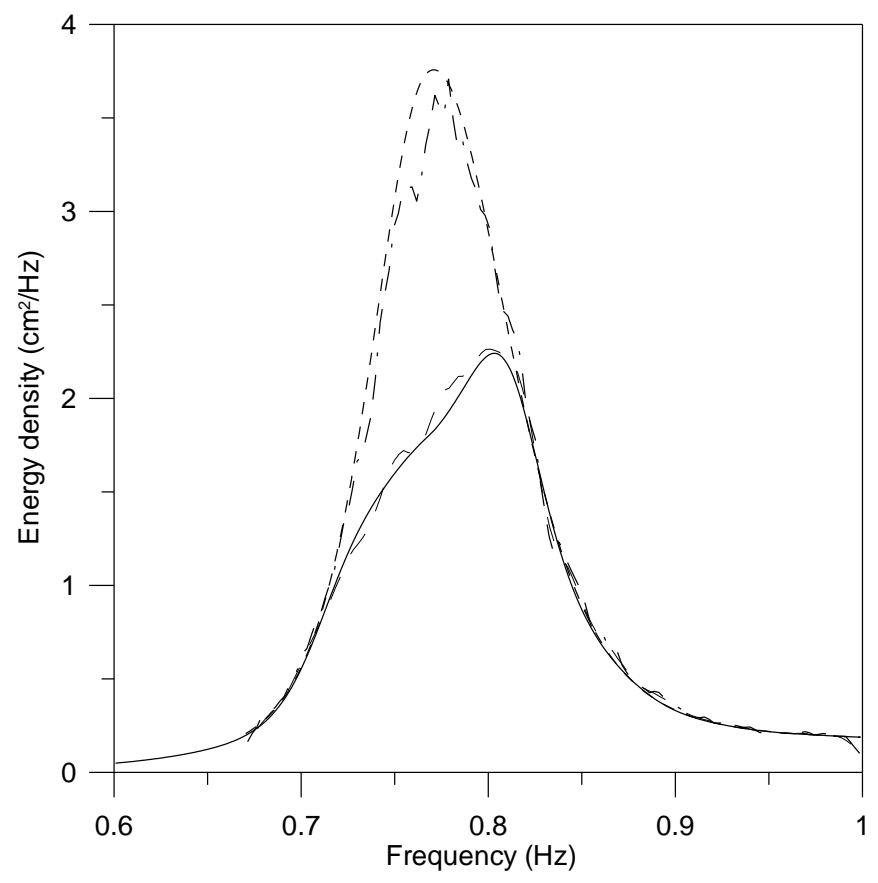

Fig. 7 Incident narrow-banded spectrum and transmitted spectra calculated by timedependent equations with weight-averaged carrier frequency; short dashed line = incident wave, solid line $=$ finite element model, long dashed line $=$ proposed model, dash-dotted line $=$ Kubo et al.'s model.

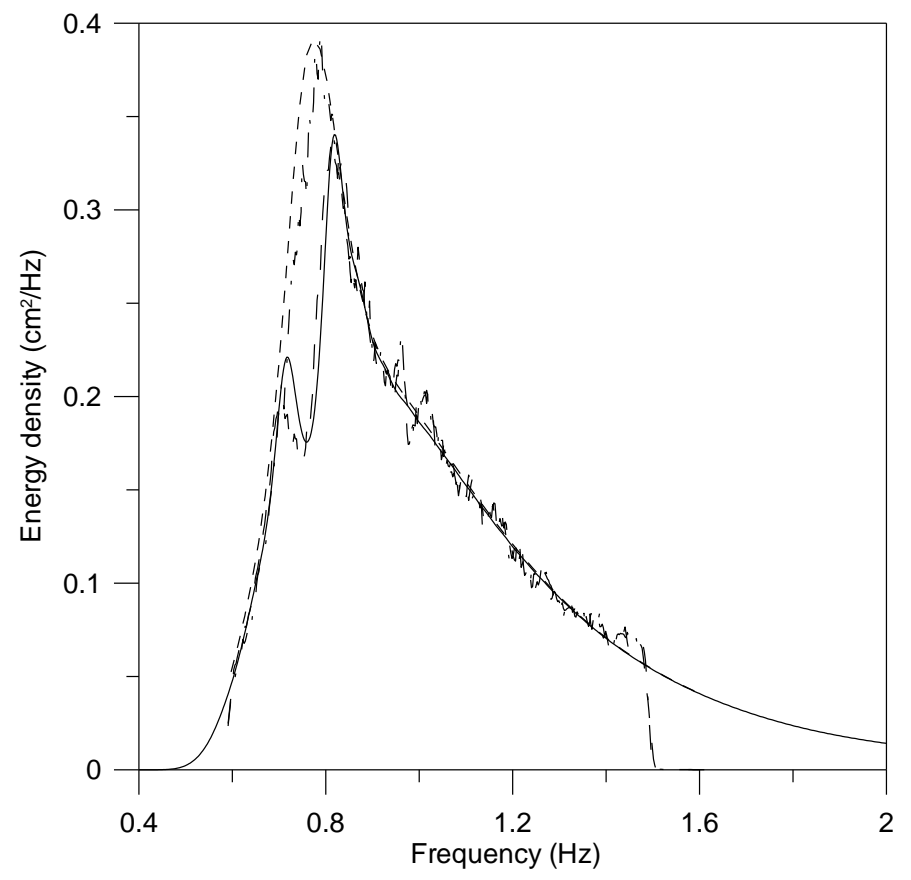

Fig. 8 Same as Fig. 7, but for broad-banded spectrum. 


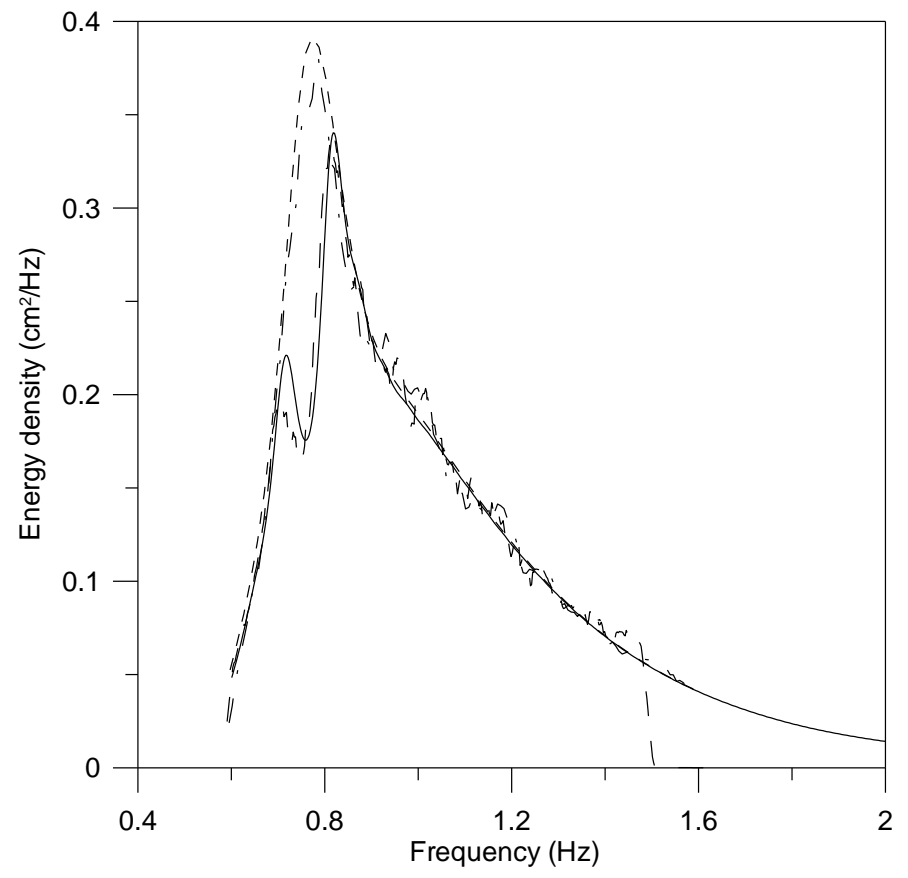

Fig. 9 Incident broad-banded spectrum and transmitted spectra calculated by timedependent equations with weight-averaged carrier frequency; short dashed line = incident wave, solid line = finite element model, long dashed line = proposed model, dash-dotted line $=$ Suh et al.'s model.

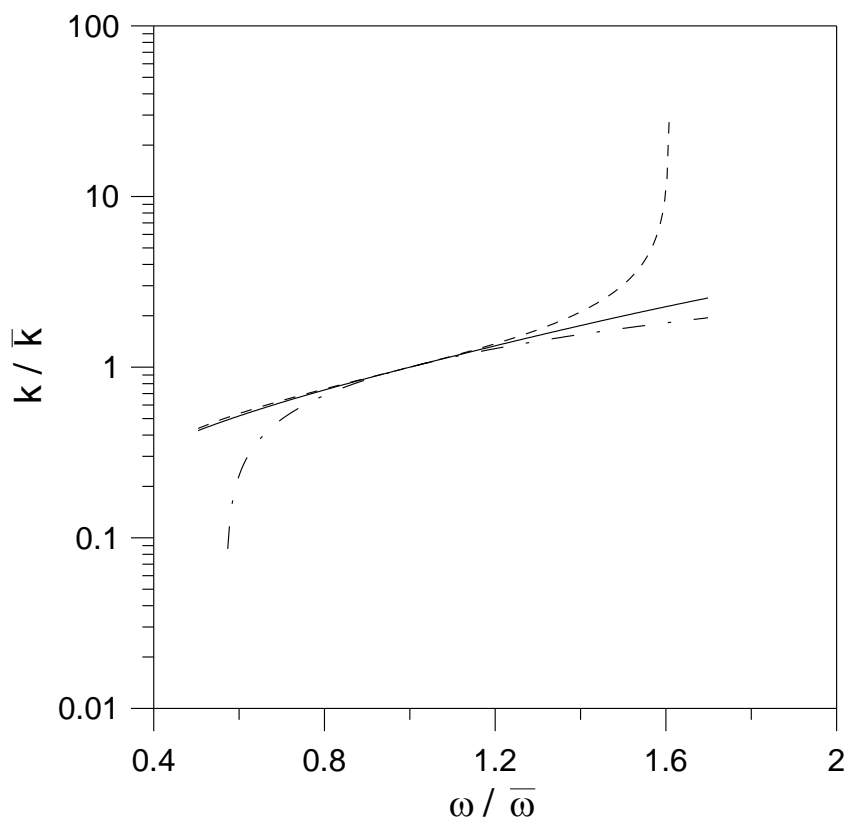

Fig. 10 Dispersion relation for $\bar{k} h=0.438 \pi$; solid line $=$ exact, dashed line $=$ proposed model, dash-dotted line $=$ Suh et al.'s model. 